\title{
Dynamic compressibility of air in porous structures at audible frequencies
}

\author{
Lafarge, Denis; Lemarinier, Pavel; Allard, Jean F.; Tarnow, Viggo
}

Published in:

Acoustical Society of America. Journal

Link to article, DOI:

$10.1121 / 1.419690$

Publication date:

1997

Document Version

Publisher's PDF, also known as Version of record

Link back to DTU Orbit

Citation (APA):

Lafarge, D., Lemarinier, P., Allard, J. F., \& Tarnow, V. (1997). Dynamic compressibility of air in porous structures at audible frequencies. Acoustical Society of America. Journal, 102(4), 1995-2006.

https://doi.org/10.1121/1.419690

\section{General rights}

Copyright and moral rights for the publications made accessible in the public portal are retained by the authors and/or other copyright owners and it is a condition of accessing publications that users recognise and abide by the legal requirements associated with these rights.

- Users may download and print one copy of any publication from the public portal for the purpose of private study or research.

- You may not further distribute the material or use it for any profit-making activity or commercial gain

- You may freely distribute the URL identifying the publication in the public portal

If you believe that this document breaches copyright please contact us providing details, and we will remove access to the work immediately and investigate your claim 


\title{
Dynamic compressibility of air in porous structures at audible frequencies
}

\author{
Denis Lafarge, Pavel Lemarinier, and Jean F. Allard \\ Laboratoire d'Acoustique associé au CNRS, URA 1101, Faculté des Sciences du Mans, Avenue Olivier \\ Messiaen, BP 535, 72017 Le Mans Cedex, France
}

Viggo Tarnow

Department of Applied Engineering Design and Production, DTU, Akademivej Bygning 358, DK 2800 Lyngby, Denmark

(Received 24 February 1996; revised 9 June 1997; accepted 16 June 1997)

\begin{abstract}
Measurements of dynamic compressibility of air-filled porous sound-absorbing materials are compared with predictions involving two parameters, the static thermal permeability $k_{0}^{\prime}$ and the thermal characteristic dimension $\Lambda^{\prime}$. Emphasis on the notion of dynamic and static thermal permeability - the latter being a geometrical parameter equal to the inverse trapping constant of the solid frame-is apparently new. The static thermal permeability plays, in the description of the thermal exchanges between frame and saturating fluid, a role similar to the viscous permeability in the description of the viscous forces. Using both parameters, a simple model is constructed for the dynamic thermal permeability $k^{\prime}(\omega)$, which is completely analogous to the Johnson et al. [J. Fluid Mech. 176, 379 (1987)] model of dynamic viscous permeability $k(\omega)$. The resultant modeling of dynamic compressibility provides predictions which are closer to the experimental results than the previously used simpler model where the compressibility is the same as in identical circular cross-sectional shaped pores, or distributions of slits, related to a given $\Lambda^{\prime}$. (C) 1997 Acoustical Society of America. [S0001-4966(97)00310-X]

PACS numbers: 43.20.Gp, 43.20.Jr, 43.55.Ev [JEG]
\end{abstract}

\section{INTRODUCTION}

In air-filled sound-absorbing media, the frequency dependence of the compressibility, which varies from the isothermal to the adiabatic value when frequency increases, plays an important role when a precise prediction of sound absorption versus frequency is needed. Beranek ${ }^{1}$ has suggested (1942) that at low frequencies the cycle of condensation and rarefaction of the enclosed air is isothermal due to the thermal exchanges between air and frame. The frequency dependence of compressibility was calculated by Zwikker and Kosten ${ }^{2}$ for pores in the form of circular cross-sectional shaped cylinders and slits. Other shapes of pores were studied later. ${ }^{3}$ It may be noticed that for cylindrical pores the compressibility depends on the shape of the cross section. For a given hydraulic radius $\bar{r}(\bar{r}$ is two times the ratio of the area to the perimeter of the cross section), this dependence can be neglected in a first approximation. In porous media with other geometries of the frame, the frequency dependence of compressibility was the same (Zwikker and Kosten, ${ }^{2}$ Attenborough, ${ }^{4}$ Allard et al. ${ }^{5}$ ) as in circular crosssectional shaped pores. The radius is related to the flow resistivity with an adjustable factor which takes into account the specificity of the geometry for the different porous frames. The adequation of such models is limited by the fact that cylindrical pores generally do not exist in porous media. A qualitative description of the effect of pore constrictions was given by Zwikker and Kosten. ${ }^{2}$ A precise description of compressibility in a pore made up of an alternating sequence of circular cross-sectional shaped cylinders was performed by Champoux and Stinson. ${ }^{6}$ An aim to relate the frequency dependence of compressibility to nonacoustical measurements was performed later by Attenborough. ${ }^{7}$ In this paper the porous material was modeled as a log-normal size distribution of tortuous slits widths. Pore size distribution can be determined nonacoustically for some materials. A parameter which characterizes the high-frequency behavior of compressibility, i.e., the thermal characteristic dimension, was identified by Champoux and Allard. ${ }^{8}$ This parameter $\Lambda^{\prime}$ is twice the ratio between the pore volume $V_{p}$ and surface $S_{p}$ and is sometimes referred to as the Kozeny radius: $\Lambda^{\prime}$ $=2 V_{p} / S_{p}$. It generalizes the notion of hydraulic radius for the case of arbitrary geometries of the frame and reduces to it for the case of cylindrical pores. This definition was inspired by the definition of the characteristic viscous dimension (Johnson et al. $\left.{ }^{9,10}\right) \Lambda$, which characterizes the viscous interaction between air and frame at high frequencies. At sufficiently high frequencies the thermal exchanges between air and frame mainly occur in a small layer close to the frame, where temperature depends on the local distance to the frame (as if the frame-air interface was plane). A normalized dynamic compressibility $\beta(\omega)$ will be used in the present work, defined by

$$
\frac{\beta(\omega)}{K_{a}}\langle p\rangle=\frac{1}{\rho_{0}}\langle\rho\rangle,
$$

where $K_{a}$ is the adiabatic bulk modulus of air, $\rho_{0}$ the air density at rest, and $\langle p\rangle$ and $\langle\rho\rangle$ the macroscopic acoustic pressure and density, respectively. (In the present paper, the symbol \langle\rangle denotes an intrinsic air-phase average.) When frequency increases, $\beta(\omega)$ tends to 
TABLE I. Viscous and thermal parameters for both porous media.

\begin{tabular}{lccccc}
\hline \hline & $\begin{array}{c}\text { Flow } \\
\text { resistivity } \\
\sigma \mathrm{Nm}^{-4} \mathrm{~s}\end{array}$ & $\begin{array}{c}\text { Viscous } \\
\text { permeability } \\
k_{0} \mathrm{~m}^{2}\end{array}$ & $\begin{array}{c}\text { Characteristic thermal } \\
\text { dimension } \Lambda^{\prime} \mathrm{m}\end{array}$ & $M^{\prime}$ & $\begin{array}{c}\text { Thermal } \\
\text { permeability } \\
k_{0}^{\prime} \mathrm{m}^{2}\end{array}$ \\
\hline Foam & 6000 & $0.3 \times 10^{-8}$ & $6.1 \times 10^{-4}$ & 0.3 & $1.3 \times 10^{-8}$ \\
Glass wool & 2300 & $0.8 \times 10^{-8}$ & $2.1 \times 10^{-3}$ & 0.03 & $1.7 \times 10^{-8}$ \\
\hline \hline
\end{tabular}

$$
\beta(\omega)=1-(\gamma-1)(1+i) \frac{\delta^{\prime}}{\Lambda^{\prime}} .
$$

In this equation, $\delta^{\prime}=\left(2 \nu^{\prime} / \omega\right)^{1 / 2}$ is the thermal skin depth (with $\nu^{\prime}=\nu / \operatorname{Pr}, \nu$ being the kinematic viscosity and $\operatorname{Pr}$ the Prandtl number), and $\gamma$ is the specific heat ratio. The imaginary part in $\beta(\omega)$ produces absorption, whereas the real part is related to the speed of sound. The characteristic dimensions $\Lambda^{\prime}$ and $\Lambda$ can be evaluated from the attenuation or the wave speed of ultrasonic pulses successively measured with different gases saturating the porous frame. ${ }^{11}$ The parameter $\Lambda^{\prime}$ can also be obtained with the BET method ${ }^{12}$ of measuring specific surfaces (pore surface $S_{p}$ for a unit mass of frame). The frame, at liquid nitrogen temperature, adsorbs a multimolecular layer of the surrounding gas (krypton can be chosen to increase the sensitivity for the case of glass wools and reticulated foams, because $S_{p}$ is much smaller than for the case of powders and materials generally measured). A measurement of the adsorbed volume of krypton provides an evaluation of $S_{p}$, and the specific volume $V_{p}$ is deduced from the two densities of the frame and the solid, or from direct measurement of the porosity and frame density. Previous measurements of $\Lambda^{\prime}$ obtained from both methods (BET and ultrasonic measurements) are in a good agreement. ${ }^{11}$

Acoustic measurements are performed on layers set on a rigid impervious baking. The frame of the porous media is always supposed to be motionless. The flow resistivity of the studied media is small (see Table I). For these materials, at low frequencies, where the transition between the isothermal and adiabatic compressibility occurs, it may be shown in the context of the Biot model ${ }^{13}$ that the frame tends to become motionless due to the weakness of the viscous forces. A simple model for the frequency dependence of compressibility consists in replacing the porous structure by an equivalent one with circular cross-sectional shaped pores having a radius equal to $\Lambda^{\prime}$. The asymptotic behavior of the predicted compressibility at high frequencies is correct. When frequency decreases, the frequency dependence of compressibility is difficult to predict, due to the increasing range of the thermal interaction between frame and air. The same problem arises for the viscous interaction at low frequencies. One approach consists in using a low-frequency parameter, the viscous static permeability related to the flow resistivity $\sigma$ by

$$
k_{0}=\eta / \sigma,
$$

where $\eta$ is the viscosity of air. The flow resistivity and the permeability are easily measurable, and when frequency tends to zero provide the essential information concerning the viscous interaction. With the parameters $\Lambda, k_{0}$, and formation factor $\alpha_{\infty} / \phi$, Johnson et al. ${ }^{10}$ have suggested a gen- eral expression for the dynamic viscous permeability $k(\omega)$ defined by

$$
\phi\langle\mathbf{v}\rangle=-\frac{k(\omega)}{\eta} \nabla\langle p\rangle
$$

where $\phi$ is the porosity (1-volumen concentration of frame material), and $\langle\mathbf{v}\rangle$ is the macroscopic velocity field. Isotropy of the porous solid is assumed for simplicity. For anisotropic structures, $k(\omega)$ is a symmetric tensor of rank two. Numerical simulations and experiments have shown that the modeling by Johnson et al. is very robust.

In the present work, a thermal analogue of the dynamic viscous permeability is defined by setting at any frequency

$$
\phi\langle\tau\rangle=\frac{k^{\prime}(\omega)}{\kappa} \frac{\partial}{\partial t}\langle p\rangle
$$

where $\langle\tau\rangle$ is the macroscopic excess temperature in air, and $\kappa$ is the coefficient of thermal conduction. The quantity $k^{\prime}(\omega)$ has the same dimensions as $k(\omega)$ (length squared). When the frame has a sufficient thermal capacity for the compressibility $\beta(\omega)$ to reach the isothermal value $\gamma$ at low frequencies, the excess temperature $\tau$ can be considered to vanish at the pore walls (this replaces the no-slip condition $\mathbf{v}=\mathbf{0}$ for viscous flow) and a static "thermal permeability" $k_{0}^{\prime}$ exists. This parameter provides the missing low-frequency thermal information and is shown to be equal to the inverse trapping constant of the porous frame (the trapping constant is defined for instance in Ref. 14 and in Appendix B). We suggest that the function $k^{\prime}(\omega)$ can be modeled in a manner similar to the permeability function $k(\omega)$ with the parameters $\Lambda^{\prime}, k_{0}^{\prime}$, and porosity $\phi$. A general connection between $k^{\prime}(\omega)$ and $\beta(\omega)$ is also established, leading to a simple expression for the compressibility function $\beta(\omega)$. Developments and justifications are reported in the different Appendices A, B, and C.

Precise and fast evaluations of compressibility are now available with a new method developed by Tarnow, ${ }^{15}$ based on measurement of the frequency and the quality factor of the quarter-wavelength resonance in a closed tube where a layer of porous material is set. Predictions with the new model are compared to measurements performed with the method developed by Tarnow.

It is to be noted that the homogenization of the viscothermal linearized acoustic equations in a gas-filled porous structure has been previously considered by SanchezPalencia and Levy. ${ }^{16}$ These authors stated the microscopic boundary value problem which enables calculating the macroscopic coefficient $\langle\tau\rangle /\langle p\rangle\left[-i \omega k^{\prime}(\omega) / \phi \kappa\right.$ in our notations] from the microstructures. However, no attempt was 
made to extract from this definition a simple modeling of the frequency dependence of compressibility. A previous attempt to generalize Johnson et al. model of dynamic permeability $k(\omega)$ to the compressibility function $\beta(\omega)$ was made by Champoux and Allard. ${ }^{8}$ However, the thermal permeability function was not considered and the role played by the inverse trapping constant $k_{0}^{\prime}$ was not apparent. The concept of thermal permeability and the resulting modeling of $\beta(\omega)$ were suggested by one of the authors in a thesis dissertation. ${ }^{17}$

\section{DYNAMIC PERMEABILITY FUNCTIONS}

\section{A. Dynamic viscous permeability}

In a porous medium, a static flow which corresponds to an averaged fluid velocity $\langle\mathbf{v}\rangle$ is related to the gradient of the averaged pressure $\boldsymbol{\nabla}\langle p\rangle$ by the Darcy law:

$$
\phi\langle\mathbf{v}\rangle=-\frac{k_{0}}{\eta} \nabla\langle p\rangle .
$$

In the frequency domain, and for disturbances characterized by a long wavelength $(\lambda \gg l$, where $l$ is typical of the pore or grain size) this static law can be replaced by

$$
\phi\langle\mathbf{v}\rangle=-\frac{k(\omega)}{\eta} \nabla\langle p\rangle,
$$

where $k(\omega)$ is the dynamic permeability. It has been shown by Johnson et al. ${ }^{10}$ (see also Avellaneda and Torquato, ${ }^{14} \mathrm{Ap}$ pendix D) that $k(\omega)$ could be replaced at high frequencies by the following asymptotic expression, analogous to (2):

$$
k(\omega)=\frac{\nu \phi}{-i \omega \alpha_{\infty}}\left[1-(1+i) \frac{\delta}{\Lambda}\right],
$$

where $\alpha_{\infty}$ is the tortuosity, $\Lambda$ is the viscous characteristic dimension, and $\delta=(2 \nu / \omega)^{1 / 2}$ is the viscous skin depth. Assuming that the three parameters, $F=\alpha_{\infty} / \phi$ (formation factor), $k_{0}$, and $\Lambda$, provide a sufficient information on the geometry, an approximation for $k(\omega)$ between the high- and the low-frequency range has been worked out by Johnson et al. ${ }^{10}$ The permeability $k(\omega)$ is the frequency response of an invariant, causal, linear system, and the poles, the zeros, and the branch points of $k(\omega)$ are located on the imaginary negative axis in the complex $\omega$ plane. Analytical functions having these properties are expected to provide fairly good approximations for $k(\omega)$ in the intermediate real frequency range, if they tend to the limits given by Eqs. (6) and (8) for small and large $\omega$. The following expression, which satisfies all the required properties, was suggested as the simplest possible ansatz:

$$
k(\omega)=k_{0} /\left[(1-(M / 2) i \widetilde{\omega})^{1 / 2}-i \widetilde{\omega}\right],
$$

where $M$ is the following dimensionless shape factor:

$$
M=\frac{8 \alpha_{\infty} k_{0}}{\phi \Lambda^{2}},
$$

and the dimensionless frequency $\widetilde{\omega}$ is defined as

$$
\widetilde{\omega}=\frac{\omega}{\nu} \frac{k_{0} \alpha_{\infty}}{\phi} .
$$

\section{B. Dynamic thermal permeability}

In a porous material having a rigid frame filled by air, a macroscopic description of the thermal exchanges between frame and air is needed to predict the compressibility of the equivalent fluid. This description will be obtained with the help of the concept of dynamical thermal permeability.

Two linearized equations which link pressure, density, and temperature variations of air considered as an ideal gas, the equation of thermal conduction and the equation of state, may be written (see for example Ref. 3)

$$
\begin{aligned}
& \kappa \nabla^{2} \tau=\frac{T_{0}}{P_{0}}\left(\rho_{0} C_{v} \frac{\partial p}{\partial t}-P_{0} C_{p} \frac{\partial \rho}{\partial t}\right), \\
& \frac{\partial p}{\partial t}=\frac{P_{0}}{\rho_{0} T_{0}}\left(\rho_{0} \frac{\partial \tau}{\partial t}+T_{0} \frac{\partial \rho}{\partial t}\right) .
\end{aligned}
$$

In these equations, $T_{0}$ and $P_{0}$ are the static temperature and pressure, and $C_{v}$ and $C_{p}$ are the specific heats (per unit mass) at constant volume and pressure, respectively. The acoustic density $\rho$ is related to the velocity $\mathbf{v}$ by

$$
\frac{\partial \rho}{\partial t}=-\rho_{0} \boldsymbol{\nabla} \cdot \mathbf{v} .
$$

Eliminating $\partial \rho / \partial t$ between Eqs. (12) and (13), and making use of the relation

$$
\frac{\rho_{0} T_{0}}{P_{0}}\left(C_{p}-C_{v}\right)=1,
$$

which is valid for ideal gas, gives, in the volume $V$ of air,

$$
\rho_{0} C_{p} \frac{\partial \tau}{\partial t}=\kappa \nabla^{2} \tau+\frac{\partial p}{\partial t} \quad(\mathbf{r} \in V)
$$

The thermal capacity and conductivity of the frame, for usual porous media, is generally very large compared to air. In a first approximation the frame can be considered as a thermostat, and the acoustical temperature $\tau$ at the contact surface $\partial V$ with the frame can be set equal to zero:

$$
\tau=0 \quad(\mathbf{r} \in \partial V) .
$$

Equation (16) and the boundary condition (17) describe the thermal diffusion in the fluid. They constitute the thermal counterpart of the linearized equations $\rho_{0}(\partial \mathbf{v} / \partial t)=\eta \nabla^{2} \mathbf{v}$ $-\boldsymbol{\nabla} p(\mathbf{r} \in V)$ and $\mathbf{v}=\mathbf{0}(\mathbf{r} \in \partial V)$ for inertial/viscous effects. [The term $(\eta+\zeta / 3) \boldsymbol{\nabla}(\boldsymbol{\nabla} \cdot \mathbf{v})$, where $\zeta$ is the second viscosity, is not written in the above Navier-Stokes equation because incompressibility of air may be assumed at the pore scale, see Appendix A, Eq. (A5c).] This suggests the definition of a "thermal permeability" $k^{\prime}(\omega)$ by means of Eq. (5), in analogy with Eq. (4). The use of the denomination "thermal permeability" is not completely appropriate, because a permeability (viscous or magnetic) is related to vectors, and the thermal permeability links two scalar quantities. Nevertheless, the designation is used to emphasize the formal similarity with the viscous permeability. In Appendix A, the firstprinciples definition of the frequency-dependent viscous and thermal permeabilities and the recipe for their calculation are derived through the generalization of the homogenization 
procedure employed by Zhou and Sheng ${ }^{18}$ in the purely viscous case.

When $\omega \rightarrow 0$ and the frame is considered as a thermostat, the excess temperature in air $\langle\tau\rangle$ is proportional to the pressure derivative $\partial\langle p\rangle / \partial t$ and a thermal Darcy's law is obtained

$$
\phi\langle\tau\rangle=\frac{k_{0}^{\prime}}{\kappa} \frac{\partial}{\partial t}\langle p\rangle .
$$

The real constant $k_{0}^{\prime}=\lim _{\omega \rightarrow 0} k^{\prime}(\omega)$ plays the role of a static thermal permeability. Under some conditions examined in Appendices $\mathrm{A}$ and $\mathrm{B}$, it is related to the trapping constant $^{14} \Gamma$ of the frame by

$$
k_{0}^{\prime}=1 / \Gamma \text {. }
$$

(The definition of $\Gamma$ is recalled in Appendix B.)

The trapping constant can be predicted from numerical simulations for simple geometries (Schwartz et al. ${ }^{19}$ ). Experimentally, nuclear magnetic resonance (NMR), which is used to characterize the geometry of porous frames (Straley et $a l .{ }^{20}$ ), could provide experimental evaluations of $\Gamma$. The excitation corresponds to the induced parallelism of nuclear spins in water. Two physical phenomena, a large deexcitation in the saturating fluid, and a noninstantaneous relaxation on contact with the frame, have prevented until now an evaluation of $\Gamma$ by NMR for plastic foams and glass wools.

When $\omega$ is large, the harmonic compressions/dilatations become adiabatic and $\rho_{0} C_{p}\langle\tau\rangle /\langle p\rangle \rightarrow 1$. More precisely, taking into account the presence of a thin thermal boundary layer at the pore walls yields ${ }^{8}$

$$
\langle\tau\rangle=\frac{\langle p\rangle}{\rho_{0} C_{p}}\left[1-(1+i) \frac{\delta^{\prime}}{\Lambda^{\prime}}\right],
$$

where it has been assumed that the pressure is a local constant - a condition justified in Appendix A-and that the temperature profile in the boundary layer is the same in the limit of high frequencies as that near a flat surface. This excludes more complicated and "fractal" pore surfaces. The asymptotic expression for $k^{\prime}(\omega)$ that can be deduced from (5) and (20) is

$$
k^{\prime}(\omega)=\frac{\nu^{\prime} \phi}{-i \omega}\left[1-(1+i) \frac{\delta^{\prime}}{\Lambda^{\prime}}\right] .
$$

The exact frequency dependence of $k^{\prime}(\omega)$ in the whole range of frequencies needs an exact description of the geometry of the frame. However, as shown in Appendix C, the function $k^{\prime}(\omega)$ verifies the same general analytical properties as $k(\omega)$. An approximate expression depending on a small number of parameters can be obtained by following the method developed by Johnson et al. described in Sec. I A. The following three parameters expression may be suggested for $k^{\prime}(\omega):^{17}$

$$
k^{\prime}(\omega)=k_{0}^{\prime} /\left[\left(1-\left(M^{\prime} / 2\right) i \widetilde{\omega}^{\prime}\right)^{1 / 2}-i \widetilde{\omega}^{\prime}\right],
$$

which involves the following (dimensionless) shape factor $M^{\prime}$ :

$$
M^{\prime}=8 k_{0}^{\prime} / \phi \Lambda^{\prime 2},
$$

and the reduced frequency

$$
\widetilde{\omega}^{\prime}=\frac{\omega}{\nu^{\prime}} \frac{k_{0}^{\prime}}{\phi}
$$

This modeling depends on three independent geometrical parameters, for instance $k_{0}^{\prime}, M^{\prime}$, and $F^{\prime}=1 / \phi$. These parameters play the same role as parameters $k_{0}, M$, and $F$ in the previous description of the viscous permeability.

\section{DYNAMIC COMPRESSIBILITY}

To describe sound propagation at the macroscopic level in rigid air-saturated material, the knowledge of two response factors will be required. One is the dynamic tortuosity

$$
\rho_{0} \alpha(\omega) \frac{\partial\langle\mathbf{v}\rangle}{\partial t}=-\nabla\langle p\rangle,
$$

obviously related to the dynamic viscous permeability $[\alpha(\omega)=-\nu \phi / i \omega k(\omega)]$. A second convenient response factor is the normalized dynamic compressibility $\beta(\omega)$, defined by Eq. (1), which can be rewritten:

$$
\frac{\beta(\omega)}{K_{a}} \frac{\partial\langle p\rangle}{\partial t}=-\nabla \cdot\langle\mathbf{v}\rangle .
$$

[This is obtained by using Eq. (14) and the identity $\langle\boldsymbol{\nabla} \cdot \mathbf{v}\rangle$ $=\boldsymbol{\nabla} \cdot\langle\mathbf{v}\rangle$, which is valid for materials having an uniform porosity; see for example Pride et al. ${ }^{21}$ and Marle. $\left.{ }^{22}\right]$ With this definition $\beta(\omega) \rightarrow 1$ when $\omega \rightarrow \infty$. Looking for plane wave solutions varying as $\exp [i(q x-\omega t)]$, Eqs. (25) and (26) yield the propagation constant and characteristic impedance of the medium

$$
\begin{aligned}
& q=\omega\left(\alpha(\omega) \beta(\omega) \frac{\rho_{0}}{K_{a}}\right)^{1 / 2}, \\
& Z=\left(\frac{\alpha(\omega)}{\beta(\omega)} \rho_{0} K_{a}\right)^{1 / 2} .
\end{aligned}
$$

Thus $\alpha$ and $\beta$ provide all pertinent information on the propagation in the medium.

The scaled compressibility $\beta(\omega)$ is directly related to the thermal permeability $k^{\prime}(\omega)$ by means of the relation

$$
\beta(\omega)=\gamma+(\gamma-1) \frac{i \omega}{\nu^{\prime}} \frac{k^{\prime}(\omega)}{\phi} .
$$

A proof is given in Appendix A. Using Eq. (22), the following simple model for frequency dependence of dynamic scaled compressibility has thus been obtained:

$$
\beta(\omega)=\gamma-(\gamma-1)\left[1+\frac{1}{-i \widetilde{\omega}^{\prime}}\left(1-\frac{M^{\prime}}{2} i \widetilde{\omega}^{\prime}\right)^{1 / 2}\right]^{-1},
$$

where the shape factor $M^{\prime}$ and reduced frequency $\widetilde{\omega}^{\prime}$ are expressed by Eqs. (23) and (24) in terms of the parameters $k_{0}^{\prime}, \Lambda^{\prime}$, and $\phi$.

It may be noticed that a similar expression for $\beta(\omega)$ was proposed by Champoux and Allard [Eq. (19) of Ref. 8]. It is, however, written in such a way that a mixing occurs between the inertial/viscous and thermal parameters, and it coincides 
with the present one only if the adjustable parameter $\sigma^{\prime}$ is set equal to $\eta \alpha_{\infty} / k_{0}^{\prime}$. The present derivation gives a physical meaning to $\sigma^{\prime}$ in Ref. 8 and shows that Eq. (19), which was extrapolated from an expression valid for cylindrical pores, is a reasonable general hypothesis for the frequency dependence of compressibility.

\section{VISCOUS AND THERMAL PERMEABILITY IN CYLINDRICAL PORES AND IN SYSTEMS OF SLITS WITH A LOG-NORMAL DISTRIBUTION FOR THE SEMI-THICKNESS}

\section{A. Cylindrical pores}

In a cylindrical pore having a cross section with dimensions much smaller than the wavelength, velocity $\mathbf{v}$ is perpendicular to the cross section, and the pressure is uniform over a cross section. Thus the viscous and thermal problem happen to have exactly the same mathematical form. This identity was noted before $e^{3,23}$ and it leads to the following obvious relations, valid for cylindrical pores:

$$
\begin{aligned}
& k^{\prime}(\omega)=k(\operatorname{Pr} \omega), \\
& k_{0}=k_{0}^{\prime}, \\
& M=M^{\prime}, \\
& \Lambda=\Lambda^{\prime}, \\
& \alpha_{\infty}=1 .
\end{aligned}
$$

A test for the model consists in predicting the compressibility with Eq. (30), and comparing with exact calculations, for circular, triangular, or rectangular cross-sectional shaped cylindrical pores. The characteristic dimensions are equal to two times the ratio of the area to the perimeter of the cross section. The viscous permeability can be calculated for the different shapes. ${ }^{24}$ Results of the exact calculations, and calculations performed with Eq. (30), are close to each other. It may be noticed that for cylindrical pores the thermal static permeability can be evaluated by measuring the flow resistivity $\sigma$

$$
\begin{aligned}
& \sigma=\eta / k_{0}, \\
& k_{0}^{\prime}=k_{0}=\eta / \sigma .
\end{aligned}
$$

\section{B. Systems of slits with a log-normal distribution for the semi-thickness}

The study, restricted to the viscous interaction, of a system of parallel slits with a log-normal distribution for the semi-thickness $b$, was initially performed by Yamamoto and Turgut. $^{25}$ This study was adapted by Attenborough ${ }^{7}$ in order to predict the dynamic tortuosity and compressibility in porous media. A brief description of the model, with further developments to predict the dependence of the characteristic dimension $\Lambda^{\prime}$ and the transition frequency for the compressibility on the parameters of the model, are given in what follows. These developments also present a nontrivial illustration of the model described in the previous sections. A distribution $e(b)$ is used, defined from the variable $w,-\infty$ $<w<\infty$, related to $b$ by $b=\exp [-w \ln 2]$, and the distribution $f$ for $w$, given by

$$
f(w)=\frac{1}{v \sqrt{2 \pi}} \exp \left[-\left(w-w_{a}\right)^{2} / 2 v^{2}\right] .
$$

The precise meaning of $e(b)$ can be deduced from Eq. (10) of Ref. 25, which gives the viscous permeability $k_{0}$ when $\alpha_{\infty}=1$

$$
k_{0}=\frac{\phi}{3} \int_{0}^{\infty} b^{2} e(b) d b
$$

Let $s(b) d b$ be the total area of slits of semi-thickness between $b$ and $b+d b$ per unit area of porous material. A direct consequence of the definition of $s(b)$ is

$$
\int_{0}^{\infty} s(b) d b=\phi
$$

The permeability can be written

$$
k_{0}=\frac{1}{3} \int_{0}^{\infty} b^{2} s(b) d b,
$$

and $s(b)$ is related to $e(b)$ by

$$
s(b)=\phi e(b) .
$$

For a unit volume of porous material, the volume of air is equal to $\phi$, and the area related to the surface of the pores in contact with air inside the volume, is twice the number $N$ of the pores, which is given by

$$
N=\int_{0}^{\infty} \frac{s(b)}{2 b} d b .
$$

The characteristic dimensions are given by

$$
\Lambda=\Lambda^{\prime}=\left[\int_{0}^{\infty} \frac{e(b)}{b} d b\right]^{-1} .
$$

They can also be obtained from Eqs. (20) and (37) of Ref. 7, which can be used to evaluate $\Lambda$ in Eq. (8) of the present paper. Equations (35) and (41) of Ref. 7 can also be used, but the right-hand sides of both equations must be multiplied by $\alpha_{\infty}$. The different methods give the same result

$$
\Lambda=\Lambda^{\prime}=2 b_{a} \exp \left[-(v \ln 2)^{2} / 2\right] .
$$

When the system of parallel slits is canted at angle $\vartheta$, the two characteristic lengths are given by the above expressions, and $k_{0}$ and $k_{0}^{\prime}$ are given by

$$
k_{0}^{\prime}=\alpha_{\infty} k_{0}=\phi\left(b_{a}^{2} / 3\right) \exp \left[2(v \ln 2)^{2}\right],
$$

where $b_{a}$ is related to $w_{a}$ by $b_{a}=\exp \left[-w_{a} \ln 2\right]$. The characteristic dimensions and the thermal permeability depend on $b_{a}$ and $v$, and not on tortuosity $\alpha_{\infty}=1 / \cos ^{2} \vartheta$. When $v=0$, these expressions give the same results as for a simple slit of semi-thickness $b_{a}$. The dynamic normalized compressibility $\beta(\omega)$ can be evaluated from the dynamic tortuosity. A simpler and equivalent evaluation can be obtained more directly by using 


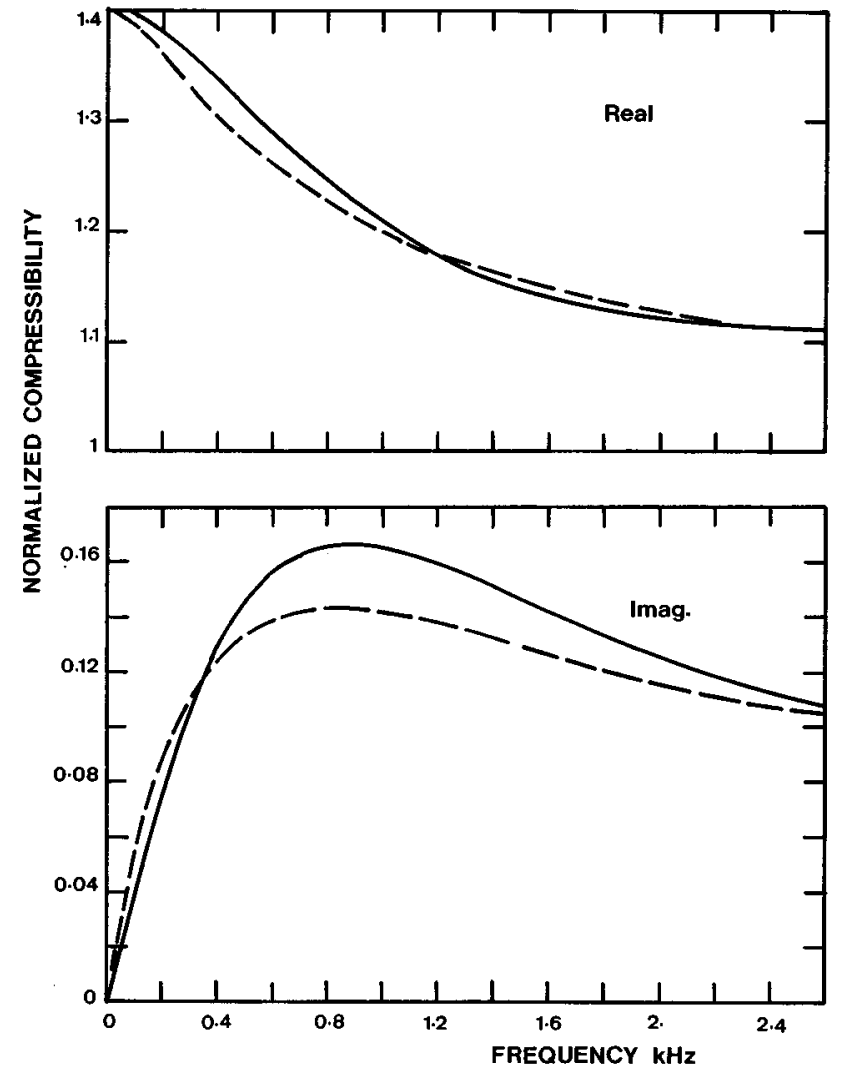

FIG. 1. Predicted normalized compressibility $\beta(\omega)$ for a slit and a system of slits, related to $\Lambda^{\prime}=0.2 \mathrm{~mm}$; slit — $----$

$$
\beta(\omega)=\int_{0}^{\infty} \beta_{b}(\omega) e(b) d b,
$$

where $\beta_{b}(\omega)$ is the compressibility in a slit having a semiwidth $b$, given by

$$
\beta_{b}(\omega)=1+(\gamma-1) \tanh \left[b\left(\frac{-i \omega}{\nu^{\prime}}\right)^{1 / 2}\right] / b\left(\frac{-i \omega}{\nu^{\prime}}\right)^{1 / 2} .
$$

The asymptotic expression of $\beta(\omega)$ when $\omega \rightarrow \infty$, compared to Eq. (2), provides the same expression for $\Lambda^{\prime}$ as Eq. (45). When $\omega \rightarrow 0$, Eq. (29) can be rewritten

$$
\beta(\omega)=\gamma+(\gamma-1) \frac{i \omega}{\nu^{\prime}} \frac{k_{0}^{\prime}}{\phi},
$$

and the asymptotic expression of $\beta(\omega)$ given by Eqs. (47) and (48) provides the same expression for $k^{\prime}(0)$ as Eq. (46). The compressibility is represented in Fig. 1 for $\Lambda^{\prime}$ $=0.2 \mathrm{~mm}$, and $v=0$ and 0.5 . In the first case the compressibility is the same as in a slit of semi-thickness $b=0.1 \mathrm{~mm}$. In the second case, $b_{a}$ and $k_{0}^{\prime}$ are given by Eqs. (45) and (46). The high-frequency representation of $\beta(\omega)$ [Eq. (2)] is already a good approximation at $2500 \mathrm{~Hz}$, for both cases. At very low frequencies, the imaginary part of $\beta(\omega)$ depends linearly on $\omega$, as indicated by Eq. (49). At last, when $v$ increases from 0 to 0.5 , the maximum for the imaginary part, at the transition frequency, is shifted toward the low frequencies. This shift is not obvious in Fig. 1, due to the broadness

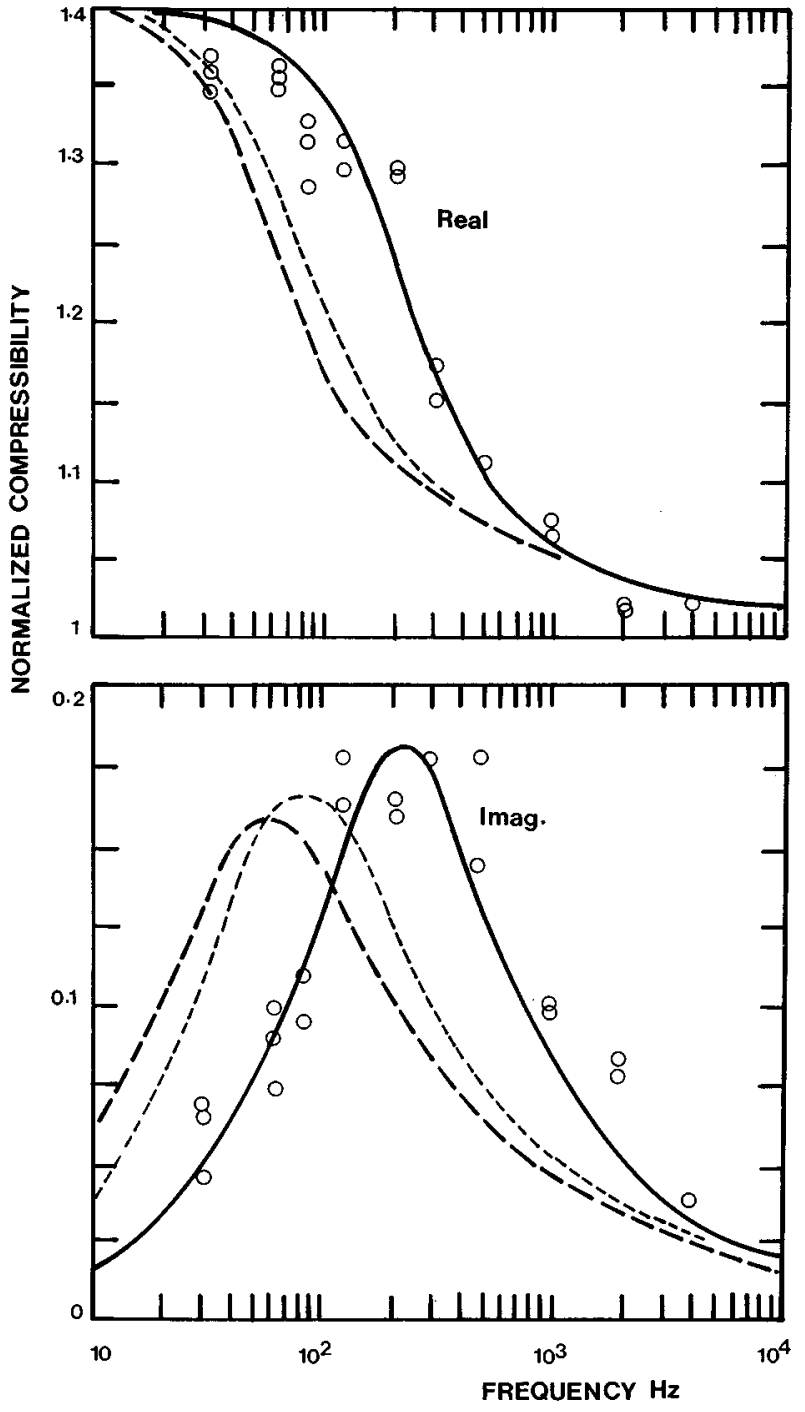

FIG. 2. Measured and predicted normalized compressibility $\beta(\omega)$ for the foam. Measurement $\bigcirc \bigcirc \bigcirc$; prediction $M^{\prime}=0.3 \longrightarrow$; prediction $M^{\prime}=1$ (circular cross-sectional shaped tube) — - - ; prediction with Attenborough's model -------.

of the peaks compared to the small value of the shift, which is close to $50 \mathrm{~Hz}$.

\section{TWO EXAMPLES}

The compressibility of air in a reticulated plastic foam and a glass wool with thin fibres of diameter close to $6 \mu \mathrm{m}$ has been evaluated with a new method which is simpler than the classical one where the acoustic impedance is measured for a layer successively backed by an impervious rigid plate, and set in front of a quarter-wavelength cavity. This new method is described in Ref. 15. The measured compressibilities are represented in Fig. 2 for the foam and Fig. 3 for the glass wool. The uncertainty of the measurements is about 0.04 for the imaginary and the real part of the compressibility at $100 \mathrm{~Hz}$. The measured values are more spread about than predicted by this uncertainty, probably due to the fact that the different samples of both materials studied cannot be characterized by identical acoustic parameters. The previous measurements by Champoux and Stinson ${ }^{6,26}$ present a 


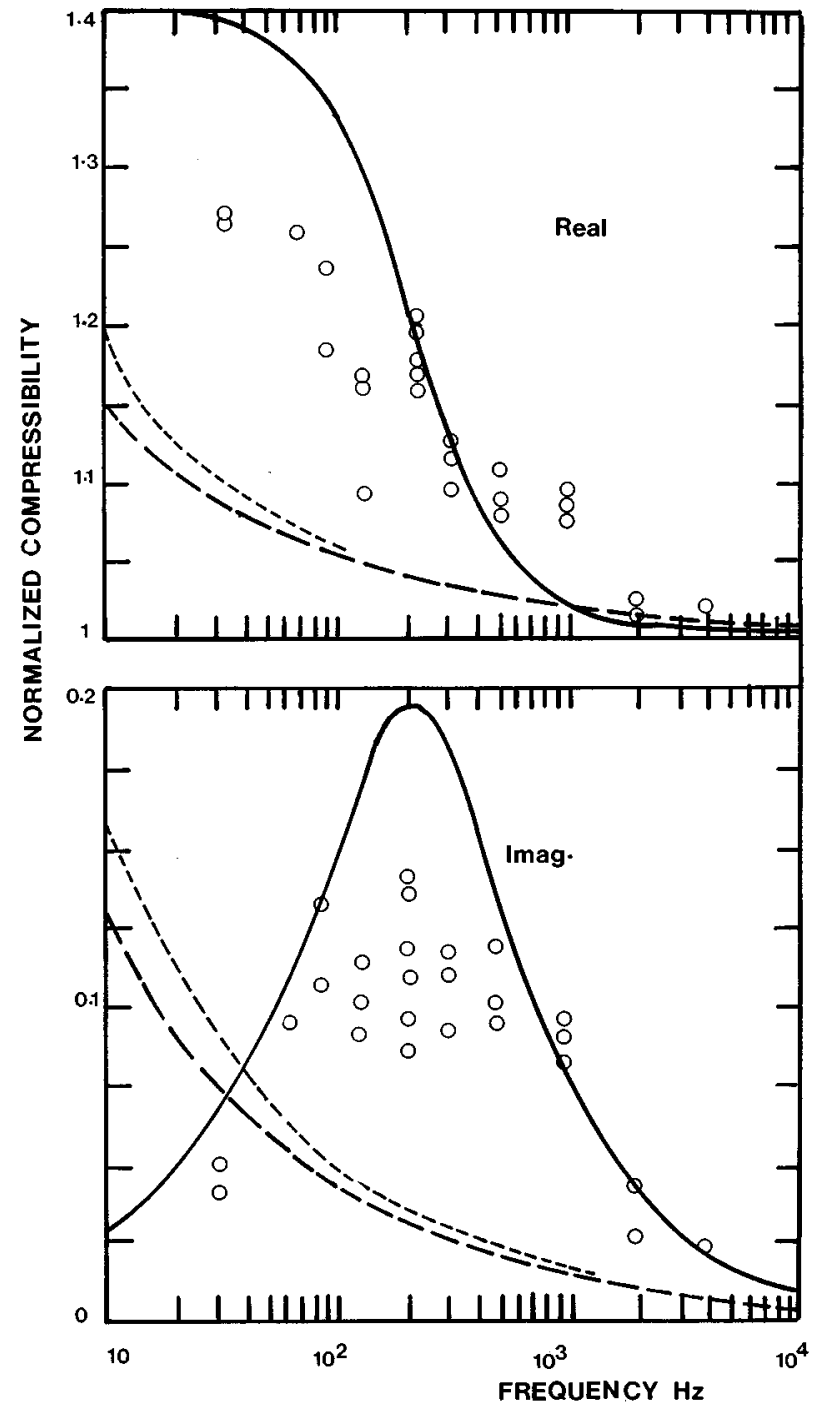

FIG. 3. Measured and predicted compressibility $\beta(\omega)$ for glass wool. Measurement $\bigcirc \bigcirc \bigcirc$; prediction $M^{\prime}=0.03 \longrightarrow$; prediction $M^{\prime}=1$ (circular cross-sectional shaped tube) _ — - ; prediction with Attenborough's model ------.

slightly smaller order of magnitude of the error. Their evaluations were obtained with a Kundt tube. Measurement method developed by one of the authors can be performed quickly, and frequencies lower than $50 \mathrm{~Hz}$ can be reached easily. The characteristic dimensions $\Lambda^{\prime}$ have been measured with the BET method described in the Introduction.

The measured dimensions $\Lambda^{\prime}$ are indicated in Table I. For both materials, the compressibility is compared with the one in a circular cross-sectional shaped pore of radius $R$ equal to $\Lambda^{\prime}$, which is obtained by setting $M^{\prime}=1$ in Eq. (30). The agreement between prediction and measurement is poor for the foam and very bad for the glass wool for the cylindrical model. An unambiguous result is that the cylindrical model with $R=\Lambda^{\prime}$ does not predict the location of the transition frequency where the imaginary part of the compressibility is maximum. The distance between the predicted and measured location is very large for glass wool, and noticeable for plastic foam. For both materials, the model based on the system of slits does not provide predictions in better agreement with measurements if $\Lambda^{\prime}$ is fixed at the measured value. For both materials, predictions obtained from the model developed by Attenborough ${ }^{7}$ have been compared with the measured compressibility. The measured tortuosity for both materials is smaller than 1.05 and the difference between the evaluations obtained from Eqs. (35) and (41) of Ref. 7, and Eq. (47) of the present work, are negligible. Equation (45) has been used to relate $v$ and $b_{a}$ with the measured value of $\Lambda^{\prime}$, and the high-frequency behavior of compressibility is the same as for the cylindrical model. For the first material, the best fit between prediction and measurement is obtained for $v=0$. The predicted compressibility is represented for $v=0, b_{a}=\Lambda^{\prime} / 2$. An increase of $v, b_{a}$, and $v$, being related by Eq. (45), does not noticeably modify the location of the maximum of the imaginary part, but simultaneously, the maximum decreases and becomes noticeably smaller than the measured value. For the second material, the predicted compressibility is also represented for $v=0$, $b_{a}=\Lambda^{\prime} / 2$. The dependence of compressibility on $v, b_{a}$, and $v$, being related by Eq. (45), is very weak, and the agreement between prediction and measurement is not improved when $v$ increases. The weak dependence of the compressibility on $v$ for frequencies higher than the transition frequency, and the behavior of the imaginary part around the transition frequency, can also be observed in Fig. 1.

As indicated in Sec. I, it has not be possible to evaluate $k_{0}^{\prime}$ nonacoustically from the measurement of the trapping constant by methods involving NMR. Without a comparison between acoustic and precise nonacoustical measurement of $k_{0}^{\prime}$, it is only possible to verify that the order of magnitude of the acoustical evaluations of $k_{0}^{\prime}$, under the hypothesis that Eq. (30) is valid, are not unphysical. Using the measured values for $\Lambda^{\prime}$, the coefficient $M^{\prime}$ has been adjusted to obtain the correct transition frequency where the imaginary part of $\beta$ is maximum. The values of the thermal permeability $k_{0}^{\prime}$ indicated in Table I have been evaluated by using Eq. (23) and the information of measured $\Lambda^{\prime}$ and fitted $M^{\prime}$. The Darcy permeabilities $k_{0}$ were obtained by independent measurements of the flow resistivity $\sigma$. The measured porosities are indicated in Table I. The method of measuring porosity is close to the one described in the book by Zwikker and Kosten. $^{2}$

With the adjusted value of $M^{\prime}$, the agreement between prediction and experience is very good for foam. It is not as good for glass wool because the fibers do not have a sufficient thermal capacity for the compressibility to reach the isothermal value at low frequencies, and the imaginary part of $\beta$ does not reach the predicted value for the maximum. In an aim of simplicity, the model has not been modified to take this effect into account. (The effect of the small thermal capacity of the frame has been studied by Tarnow. ${ }^{27}$ See Appendix B for a simple criterion which ensures that this effect is small.) The important fact for the glass wool is that the transition frequency predicted by the one parameter model (with this parameter being equal to the characteristic length $\Lambda^{\prime}$ ) is very far from the actual frequency. This is related to the very small value of $M^{\prime}$, which must be equal to one if the circular cross-sectional shaped model is valid. The volume of fiber per unit volume of glass wool is very small, 
about 1/1000. A previous study ${ }^{28}$ on periodic lattices of spheres indicates that the modeling of the viscous permeability by Eq. (9) is robust, but $M$ becomes very small at low concentrations. The same trend is observed experimentally for $M^{\prime}$. In fact, exact calculations of the various parameters are not difficult to perform for regular arrays of cylinders in a dilute limit and the mentioned behavior $M, M^{\prime} \rightarrow 0$ with $\phi \rightarrow 1$ can be checked explicitly. ${ }^{29}$ For identical circular pores and slits, $M^{\prime}=1$ and 0.66, respectively. For the lognormal distribution, $M^{\prime}$ increases with $v$ and is always larger than 0.66 . These representations cannot be used when $M^{\prime}$ $\ll 1$ to predict compressibility.

By inspection of the Johnson et al. function (9) it may be observed that in a limit $M \rightarrow 0$, a simple, one relaxation time, "Debye" scaling ${ }^{30}$ occurs, $k / k_{0} \rightarrow 1 /(1-i \widetilde{\omega})$, as long as the frequency fulfills $M \widetilde{\omega} \ll 1$-which is the case for most of the relaxational pattern. Disregarding effects related to the finite thermal capacity of the solid, the same trend will occur for the thermal quantities, and we may expect that for small $M^{\prime}$ a "Debye" scaling will apply to $k^{\prime}$ (as long as $M^{\prime} \widetilde{\omega}^{\prime}$ $\ll 1)$. In terms of the compressibility $\beta$ this means that we should, in this limit, obtain $\beta \approx \gamma-(\gamma-1)\left[-i \widetilde{\omega}^{\prime} /(1\right.$ $\left.\left.-i \widetilde{\omega}^{\prime}\right)\right]$. It can be easily verified that this simple form of $\beta$ could have been used in Fig. 3 in place of the given two parameters prediction $\left(M^{\prime}=0.03, \Lambda^{\prime}=2.1 \times 10^{-3} \mathrm{~m}\right)$.

The above simple form for $\beta$ can be used to recover in a more direct manner the given value $k_{0}^{\prime}=1.7 \times 10^{-8} \mathrm{~m}^{2}$ of the thermal permeability for glass wool. The characteristic frequency $f_{c}$ where $\operatorname{Im}(\beta)$ is maximum corresponds in this case to $\widetilde{\omega}^{\prime}=1$, i.e., the thermal permeability can be estimated by means of the relation $k_{0}^{\prime}=\nu^{\prime} \phi /\left(2 \pi f_{c}\right)$. With $f_{c} \approx 200 \mathrm{~Hz}$, as indicated by the measurements in Fig. 3, we obtain $k_{0}^{\prime}$ $=1.68 \times 10^{-8} \mathrm{~m}^{2}$ in agreement with the previous result. For glass wool the simplified Debye expression for $\beta$ related to very small concentrations can be used to evaluate $k_{0}^{\prime}$.

For foam, the acoustically measured thermal permeability is larger than the viscous permeability by a factor close to 4. This factor is equal to 2 for glass wool. Like glass wool, reticulated foam can be considered a fibrous material. It may be noticed that a fibrous material having parallel fibers, in a flow parallel to the fibers, can be considered as one pore limited by the surface of the fibers. In that case, the thermal and the viscous permeability are equal. If the flow is perpendicular to the fibers, the thermal permeability is the same as in the previous case, but the viscous permeability is approximately decreased by a factor of $2 .{ }^{23}$ In fact, an exact calculation for regular arrays of cylinders in a dilute limit predicts this factor of $2 .{ }^{29}$ The fibers in the porous foam are not parallel and their sections are more like triangles than circles. We do not know whether taking into account these details on the structure yields the desired factor of 4 for foam.

\section{CONCLUSION}

The present study presents a simple extension to thermal effects of an approach worked out by Johnson et al. for viscous effects. This modeling uses two parameters for predicting the dynamic compressibility of air in reticulated foams and glass wools. The thermal characteristic dimension and the thermal permeability have been used, which characterize the thermal exchanges between air and frame in the highand low-frequency range, respectively. Due to the complexity of the frame of these materials, it has not been possible to compare the acoustically evaluated static permeability with predicted values. Comparison with measurements of related quantities like the trapping constant would be an interesting complement to the present study.

\section{ACKNOWLEDGMENT}

The authors thank A. Gedeon (Laboratoire de Chimie des Surfaces, University of Paris VI) for the measurements of the specific surfaces.

\section{APPENDIX A: MICROSTRUCTURAL APPROACH}

Under the excitation of an external harmonic source with frequency $\omega$, the air motion inside the porous material is completely characterized, at the microscopic level, by values of the velocity $\mathbf{v}(\mathbf{r}, \omega)$, excess pressure $p(\mathbf{r}, \omega)$, and excess temperature $\tau(\mathbf{r}, \omega)$. In the framework of linearized acoustics, air being considered as an ideal gas, these variables obey the following coupled equations in the porous domain $V$ :

$$
\begin{aligned}
& -i \omega \rho_{0} \mathbf{v}=-\nabla p+\eta \nabla^{2} \mathbf{v}+(\eta / 3+\zeta) \nabla(\nabla \cdot \mathbf{v}), \\
& -i \omega \frac{1}{P_{0}}\left(p-\left(P_{0} / T_{0}\right) \tau\right)=-\nabla \cdot \mathbf{v}, \\
& -i \omega \rho_{0} C_{p} \tau=-i \omega p+\kappa \nabla^{2} \tau
\end{aligned}
$$

The boundary conditions at the contact surface with the frame are

$$
\mathbf{v}=\mathbf{0}, \quad(\mathbf{r} \in \partial V)
$$

and

$$
\tau=0, \quad(\mathbf{r} \in \partial V) .
$$

The general solution of Eqs. (A1a)-(A1d) for any nontrivial frame geometry would be difficult, if not impossible. For instance, significant coupling effects between viscous and thermal effects can occur if the porous material contains cavities which are weakly coupled with the main pores. Nevertheless, for the materials studied, i.e., air-filled glass wools and reticulated foams, the frame is made of fibers which occupy a small part of the volume, and the interplay between viscous and thermal effects can be neglected in a first approximation. One may identify a dimensionless small parameter $\epsilon$ in the problem that would enable significant simplifications for the viscous permeability and thermal permeability calculation. Like in the work by Zhou and Sheng ${ }^{18}$ this small parameter is defined as the ratio between two length scales, $\epsilon=l / L$, in which the small scale $l$ is given by the typical pore size, and the large scale $L$ is given by the product of the air sound speed $c=\sqrt{K_{a} / \rho_{0}}$ and an intrinsic viscous relaxation time $\vartheta_{v}=l^{2} / \nu$. The characteristic frequency $2 \pi / \vartheta_{v}$ is a rough estimate of a viscous rollover frequency where, when $\omega$ increases, a transition occurs between a viscous dominated regime and an inertia dominated regime. The Prandtl number $\operatorname{Pr}$ is of order $1(\sim 0.71$ for air) and the thermal rollover frequency where a transition occurs between 
isothermal and adiabatic regimes, is expected to be on the same order as the viscous rollover frequency. For air saturating usual acoustic materials with $l \approx 10^{-4} \mathrm{~m}, \epsilon$ is on the order of $10^{-4}$.

By expressing time in units of $\vartheta_{v}$ and lengths in units of $l$, Eqs. (A1a)-(A1d) may be written in dimensionless form

$$
\begin{aligned}
& -i \omega \mathbf{v}=-\frac{1}{\epsilon} \boldsymbol{\nabla} p+\nabla^{2} \mathbf{v}+\left(\frac{1}{3}+\frac{\zeta}{\eta}\right) \boldsymbol{\nabla}(\boldsymbol{\nabla} \cdot \mathbf{v}), \\
& -i \omega\left(p-\frac{\gamma-1}{\gamma} \operatorname{Pr} \tau\right)=\frac{-1}{\gamma \epsilon} \boldsymbol{\nabla} \cdot \mathbf{v}, \\
& -i \omega \operatorname{Pr} \tau=-i \omega p+\nabla^{2} \tau, \\
& \mathbf{v}=\mathbf{0} \quad(\mathbf{r} \in \partial V), \\
& \tau=0 \quad(\mathbf{r} \in \partial V) .
\end{aligned}
$$

Here, $p$ is in units of $L \eta^{2} / \rho_{0} l^{3}$ following the assumption that the macroscopic pressure gradient should be on the same order as $\eta v / l^{2}$ in accordance with Darcy's law (6). Similarly, $\tau$ is in units of $L \eta^{3} / \rho_{0}^{2} \kappa l^{3}$ following the assumption that the pressure derivative $\partial p / \partial t$ should be on the same order as $\kappa \tau / l^{2}$ in accordance with the thermal analogue of Darcy's law (18). As in the Zhou and Sheng treatment, ${ }^{18}$ these assumptions are justified by our consistent derivation of the two "Darcy's laws" from Eqs. (A2a) to (A2e). The following thermodynamic identities were used in writing Eqs. (A2b) and (A2c):

$$
\begin{aligned}
& \frac{P_{0}}{T_{0} \rho_{0} C_{p}}=\frac{\gamma-1}{\gamma}, \\
& \kappa=\frac{\eta C_{p}}{\operatorname{Pr}} .
\end{aligned}
$$

A brief discussion of the validity of boundary condition (A2e) is given in Appendix B.

The existence of a small parameter $\epsilon$ associated with rapid fluctuations in the structure of the air-filled porous domain can now be taken into account using the well-known method of homogenization. We assume that the different fields depend on two scales: a slow scale $\mathbf{x}=\mathbf{r} / L$ and a fast scale $\mathbf{y}=\mathbf{x} / \boldsymbol{\epsilon}=\mathbf{r} / l$. We shall derive the global (macroscopic) equations governing sound propagation using a two-scale expansion

$$
\begin{aligned}
& \mathbf{v}(\mathbf{x}, \mathbf{y})=\mathbf{v}_{0}(\mathbf{x}, \mathbf{y})+\epsilon \mathbf{v}_{1}(\mathbf{x}, \mathbf{y})+\cdots, \\
& p(\mathbf{x}, \mathbf{y})=p_{0}(\mathbf{x}, \mathbf{y})+\epsilon p_{1}(\mathbf{x}, \mathbf{y})+\cdots, \\
& \tau(\mathbf{x}, \mathbf{y})=\tau_{0}(\mathbf{x}, \mathbf{y})+\epsilon \tau_{1}(\mathbf{x}, \mathbf{y})+\cdots, \\
& \boldsymbol{\nabla}=\epsilon \boldsymbol{\nabla}_{x}+\boldsymbol{\nabla}_{y} .
\end{aligned}
$$

Substituting (A4a)-(A4d) into (A2a)-(A2e) and collecting powers of $\epsilon$ yields the leading order equations

$$
\begin{aligned}
& \boldsymbol{\nabla}_{y} p_{0}=0, \\
& -i \omega \mathbf{v}_{0}=-\nabla_{x} p_{0}-\nabla_{y} p_{1}+\nabla_{y}^{2} \mathbf{v}_{0}, \\
& \boldsymbol{\nabla}_{y} \cdot \mathbf{v}_{0}=0,
\end{aligned}
$$

$$
\begin{aligned}
& i \omega\left(p_{0}-\frac{\gamma-1}{\gamma} \operatorname{Pr} \tau_{0}\right)=\frac{1}{\gamma}\left(\nabla_{x} \cdot \mathbf{v}_{0}+\nabla_{y} \cdot \mathbf{v}_{1}\right), \\
& -i \omega \operatorname{Pr} \tau_{0}=-i \omega p_{0}+\nabla_{y}^{2} \tau_{0} .
\end{aligned}
$$

These equations are supplemented by absorbing conditions on the air-solid interface

$$
\begin{aligned}
& \mathbf{v}_{0}=0, \quad \mathbf{v}_{1}=0, \ldots \quad(\mathbf{r} \in \partial V), \\
& \tau_{0}=0, \ldots . \quad(\mathbf{r} \in \partial V) .
\end{aligned}
$$

Equation (A5a) shows that $p_{0}$, independent of coordinate $\mathbf{y}$, may be regarded as the externally applied pressure. Equations (A5e), (A5a), and (A5g) then indicate that, to the lowest order, the temperature field originates from a spatially uniform, harmonic, heating in the air domain, with perfect absorbing conditions on the solid boundaries. Equation (A5c) indicates that to the lowest order, the velocity field may be regarded as incompressible on the $\mathbf{y}$ scale.

The fields $p_{1}, \mathbf{v}_{0}$, and $\tau_{0}$ are solutions to two independent set of linear equations, Eqs. (5c), (A5d), and (A5f) on one hand, and Eqs. (A5d) and (A5g) on the other hand. Hence, they may be formally expressed as linear operators acting on the source terms $-\nabla_{x} p_{0}$ and $-i \omega p_{0}$, i.e.,

$$
\begin{aligned}
& p_{1}=\mathbf{P}(\mathbf{x}, \mathbf{y}) \cdot\left(-\nabla_{x} p_{0}\right),
\end{aligned}
$$

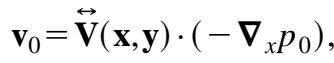

$$
\begin{aligned}
& \tau_{0}=\Theta(\mathbf{x}, \mathbf{y})\left(-i \omega p_{0}\right) .
\end{aligned}
$$

The linear operators $\Theta, \mathbf{P}$, and $\overleftrightarrow{\mathbf{V}}$ are, respectively, scalar, vector, and second rank tensor operators. By substituting Eqs. (A6a)-(A6c) into the two independent set noted above, we get the generic equations satisfied by the operators $\mathbf{P}, \overleftrightarrow{\mathbf{V}}$, and $\Theta$

$$
\begin{aligned}
& \nabla_{y} \mathbf{P}-i \omega \overleftrightarrow{\mathbf{V}}-\nabla_{y}^{2} \overleftrightarrow{\mathbf{V}}=\overleftrightarrow{\mathbf{I}}, \\
& \nabla_{y} \cdot \overleftrightarrow{\mathbf{V}}=\mathbf{0}, \\
& \overleftrightarrow{\mathbf{V}}=\overleftrightarrow{\mathbf{0}} \quad(\mathbf{r} \in \partial V)
\end{aligned}
$$

and

$$
\begin{aligned}
& -i \omega \operatorname{Pr} \Theta-\nabla_{y}^{2} \Theta=I, \\
& \Theta=0 \quad(\mathbf{r} \in \partial V) .
\end{aligned}
$$

In these equations $\overleftrightarrow{\mathbf{I}}$ and $I$ are the unit second rank tensor and unit scalar operators $\left[(\stackrel{\leftrightarrow}{\mathbf{I}})_{i j}=I \delta_{j j}\right.$ where $\delta_{i j}$ is the Kronecker symbol]. Given the solutions $\mathbf{V}$ and $\Theta$, the dynamic viscous and thermal permeabilities $k(\omega)$ and $k^{\prime}(\omega)$ may be directly calculated by averaging $\mathbf{v}_{0}$ and $\tau_{0}$ over the $y$ variable

$$
\begin{aligned}
& \left\langle\mathbf{v}_{0}\right\rangle_{y}=\langle\overleftrightarrow{\mathbf{V}}(\mathbf{x}, \mathbf{y})\rangle_{y} \cdot\left(-\nabla_{x} p_{0}\right), \\
& \left\langle\tau_{0}\right\rangle_{y}=\langle\Theta(\mathbf{x}, \mathbf{y})\rangle_{y}\left(-i \omega p_{0}\right) .
\end{aligned}
$$

Comparing Eqs. (A9) and (A10) with the two dynamic Darcy's laws (4) and (5), we get

$$
\stackrel{\leftrightarrow}{k}(\omega)=\phi\langle\overleftrightarrow{\mathbf{V}}(\mathbf{x}, \mathbf{y})\rangle_{y}
$$

as the definition of the permeability tensor, and 


$$
k^{\prime}(\omega)=\phi\langle\Theta\rangle_{y}
$$

as the definition of the (scalar) thermal permeability. The dimensionless permeabilities given by (A11) and (A12) must be multiplied by $l^{2}$ to recover the dimensionalized quantities in Eqs. (4) and (5).

The remaining microscopic equation (A5d) may serve to establish the general connexion (29) between thermal permeability and compressibility. Averaging (A5d) gives

$$
i \omega\left(p_{0}-\frac{\gamma-1}{\gamma} \operatorname{Pr}\left\langle\tau_{0}\right\rangle_{y}\right)=\frac{1}{\gamma}\left(\boldsymbol{\nabla}_{x} \cdot\left\langle\mathbf{v}_{0}\right\rangle_{y}+\left\langle\boldsymbol{\nabla}_{y} \cdot \mathbf{v}_{1}\right\rangle_{y}\right) .
$$

The second term on the right-hand side of (A13) is now shown to be zero. Let $V_{R}$ be a large sphere of radius $R$ centered at the origin. Then

$$
\left\langle\boldsymbol{\nabla}_{y} \cdot \mathbf{v}_{1}\right\rangle_{y}=\frac{1}{V_{R}} \int_{V_{R}} \boldsymbol{\nabla}_{y} \cdot \mathbf{v}_{1}(\mathbf{x}, \mathbf{y}) d \mathbf{y}=\frac{1}{V_{R}} \int_{\partial V_{R}} \mathbf{v}_{1} \cdot \mathbf{n} d \mathbf{y} .
$$

The surface $\partial V_{R}$ consists of two parts: $\left(S_{R}\right)_{i}$ is the part of the pore surface within $V_{R}$, and $\left(S_{R}\right)_{e}$ is the part of the surface of the large sphere within the pore domain. Using the boundary condition $\mathbf{v}_{1}=\mathbf{0}$ on $\left(S_{R}\right)_{i}$ we have

$$
\left\langle\nabla_{y} \cdot \mathbf{v}_{1}\right\rangle_{y}=\frac{1}{V_{R}} \int_{\left(S_{R}\right)_{e}} \mathbf{v}_{1} \cdot \mathbf{n} d \mathbf{y},
$$

and letting $R \rightarrow \infty$ we finally arrive at

$$
\left\langle\nabla_{y} \cdot \mathbf{v}_{1}\right\rangle_{y}=0 \text {. }
$$

By using Eq. (A14) and Eqs. (A10) and (A12), the averaged equation (A13) can then be rewritten

$$
-\nabla_{x} \cdot\left\langle\mathbf{v}_{0}\right\rangle_{y} /-i \omega p_{0}=\gamma+(\gamma-1) \operatorname{Pr} \frac{k^{\prime}(\omega)}{\phi} i \omega .
$$

This ratio represents the dimensionless, frequency-dependent compressibility function $\beta(\omega)$. [Reintroducing dimensionalized variables on the left-hand side of Eq. (A15) a factor $L \widetilde{\omega} \widetilde{v} \widetilde{v}$ appears where tilded quantities are reference quantities. Using the identifications $\tilde{p}=L \eta^{2} / \rho_{0} l^{3}, \quad L$ $=\sqrt{K_{a} / \rho_{0}}\left(l^{2} / \nu\right)$, and $\tilde{v}=\widetilde{\omega} l$, it may be checked that this factor coincide with the adiabatic bulk modulus $K_{a}$.] Reintroducing dimensionalized variables on the right-hand side of Eq. (A15), the product $k^{\prime}(\omega) \omega$ must be written $\left[k^{\prime}(\omega) / l^{2}\right]$ $\times\left(\omega l^{2} / \nu\right)$ and the final result is

$$
\beta(\omega)=\gamma+(\gamma-1) \frac{i \omega}{\nu^{\prime}} \frac{k^{\prime}(\omega)}{\phi},
$$

with $\nu^{\prime}=\nu / \operatorname{Pr}$.

\section{APPENDIX B: RELATION WITH DIFFUSION- CONTROLLED REACTIONS}

\section{Trapping constant}

The trapping constant can be defined as follows. Inside the fluid that saturates the frame, an excitable solute is created with a spatially constant density $s$. The solute diffuses with a diffusion constant $D$, and is instantly relaxed on contact with the frame. The equations governing the diffusioncontrolled process are

$$
\begin{aligned}
& \frac{\partial u}{\partial t}-D \nabla^{2} u=s \quad(\mathbf{r} \in V) \\
& u=0 \quad(\mathbf{r} \in \partial V),
\end{aligned}
$$

where $u$ is the concentration of solute per unit volume of saturating fluid. At equilibrium, $\partial u / \partial t=0$, and the trapping constant is given by

$$
\Gamma=s /(\langle u\rangle \phi D) \text {. }
$$

The trapping constant is related to the mean survival time $\theta$ of solute particles by

$$
\theta=1 / \Gamma \phi D \text {. }
$$

Application of Eq. (B3) gives

$$
\theta=\langle u\rangle / s \text {. }
$$

\section{Frequency-dependent mean survival time and relation with thermal permeability}

A similar survival problem can be defined in harmonic regime when the excitable solute is created with a spatially constant density $s e^{-i \omega t}$. The frequency-dependent mean survival time is defined by (Torquato and Avellaneda ${ }^{31}$ )

$$
\theta(\omega)=\langle u(\omega)\rangle / s,
$$

where $u(\omega)$ solves the diffusion-controlled problem

$$
\begin{aligned}
& -i \omega u-D \nabla^{2} u=s \quad(\mathbf{r} \in V), \\
& u=0 \quad(\mathbf{r} \in \partial V),
\end{aligned}
$$

where $s$ is a spatial constant. Note that $\theta(0)$ is just the standard static survival time (B4).

A comparison between Eqs. (B7) and (B8) and the thermal diffusion equations Eqs. (16) and (17) shows identical problems (with substitutions $s=-i \omega p / \rho_{0} C_{p}, \quad u=\tau, \quad D$ $\left.=\nu^{\prime}=\kappa / \rho_{0} C_{p}\right)$ provided the pressure $p$ can be considered as a spatial constant at the pore scale. It was noted in Appendix A that this constancy follows from the simple two-scale analysis [see Eq. (A5a)] and that it is a reasonable hypothesis for the materials studied. Hence, the following identity is obtained:

$$
k^{\prime}(\omega)=\nu^{\prime} \phi \theta(\omega),
$$

where $\theta(\omega)$ corresponds to $D=\nu^{\prime}$. Setting $\omega=0$ in Eq. (B9) and using (B4) yields relation (19) between static thermal permeability $k_{0}^{\prime}$ and trapping constant $\Gamma$.

\section{Condition on the heat capacity of the porous solid}

So far we have assumed the validity of the boundary conditions $\tau=0$ on the air-solid interface. A static thermal permeability exists and an isothermal low-frequency limit for the compressibility $\lim _{\omega \rightarrow 0} \beta(\omega)=\gamma$ is obtained by using Eq. (A16). This assumption is justified only in a limit where the ratio

$$
r=\frac{\left(\rho_{0} C_{p}\right)_{\mathrm{air}} \phi}{\left(\rho_{0} C_{p}\right)_{\text {solid }}(1-\phi)}
$$

of the heat capacity coefficients is small: $r \ll 1$. For usual porous foams this ratio is on the order of $1 / 20$. However, 
greater values can be found in the case of light glass wools. The effect of finite heat capacity of the solid on the lowfrequency limit of $\beta(\omega)$ can be predicted by means of simple calorimetric considerations. In a quasi-static limit for compressions and dilatations, the excess temperature has sufficient time to equalize in the air and solid part. Thus in the limit $\omega=0$ we may write

$$
\left[\left(\rho_{0} C_{p}\right)_{\text {air }} \phi+\left(\rho_{0} C_{p}\right)_{\text {solid }}(1-\phi)\right] \frac{\partial\langle\tau\rangle}{\partial t}=\phi \frac{\partial\langle p\rangle}{\partial t} .
$$

A comparison between Eqs. (5) and (B11) shows that in this quasi-static limit $k^{\prime}(\omega)$ behaves like $\nu^{\prime} \phi r /-i \omega(1+r)$. The general relation (A16) between $k^{\prime}(\omega)$ and $\beta(\omega)$ still applies to this case because the boundary conditions $\tau=0$ were not explicitly used. Making use of this connection finally yields

$$
\lim _{\omega \rightarrow 0} \beta(\omega)=\frac{\gamma+r}{1+r}
$$

Equation (B12) shows that the effect of finite heat capacity of the solid can be ignored when $r \ll 1$ (this is the mentioned criterion in Sec. IV). The boundary conditions $\tau=0$ then lead to reasonable results and a static thermal permeability may be assumed to exist.

\section{APPENDIX C: SINGULARITIES OF THERMAL RESPONSE FUNCTIONS}

As shown by Johnson et al. ${ }^{10}$ (Appendix A), the two response functions $k(\omega)$ and $\alpha(\omega) \equiv \nu \phi /-i \omega k(\omega)$ are analytic functions of $\omega$ everywhere in the complex plane except for values of $\omega$ on the negative imaginary axis. Thus any pole, branch point, or zero of these functions lies on the imaginary axis. We wish to point out that the same general properties apply to the thermal functions $k^{\prime}(\omega)$ and $\alpha^{\prime}(\omega)$ $\equiv \nu^{\prime} \phi /-i \omega k^{\prime}(\omega)$.

The definitions of $k^{\prime}(\omega)$ and $\alpha^{\prime}(\omega)$ are

$$
\begin{aligned}
& \phi\langle\tau\rangle=\frac{k^{\prime}(\omega)}{\kappa} \frac{\partial}{\partial t}\langle p\rangle, \\
& \rho_{0} C_{p} \alpha^{\prime}(\omega) \frac{\partial}{\partial t}\langle\tau\rangle=\frac{\partial}{\partial t}\langle p\rangle,
\end{aligned}
$$

where $\tau$ and $p$ verify the local equations

$$
\begin{aligned}
& \rho_{0} C_{p} \frac{\partial \tau}{\partial t}=\kappa \nabla^{2} \tau+\frac{\partial p}{\partial t}, \quad(\mathbf{r} \in V), \\
& \boldsymbol{\nabla} p=\mathbf{0} \quad(\mathbf{r} \in V), \\
& \tau=0 \quad(\mathbf{r} \in \partial V) .
\end{aligned}
$$

[Equation (C4) is written because of Eq. (A5a).]

In order to study the singularities of the functions $k^{\prime}(\omega)$ and $\alpha^{\prime}(\omega)$, we imagine a volume of the porous sample enclosed by a thin membrane, which is allowed to be moving through the solid without disturbing it, while being impervious to the fluid. It is assumed that the membrane behaves as an adiabatic wall (Woods ${ }^{32}$ ). The singularities of response factors are related to unforced oscillations of the system, and we shall prove that the latter are purely damped. Due to Eq.
(C1), a pole of $k^{\prime}(\omega)$ means that there exists a nontrivial solution to the above microscopic equations, having the property that $p=0$. This occurs if the membrane is a pressure-release surface. The pressure term in (C3) is not present, and we are left with the Fourier equation of heat diffusion

$$
\rho_{0} C_{p} \frac{\partial \tau}{\partial t}=\kappa \nabla^{2} \tau
$$

The oscillations $\exp (-i \omega t)$ of the system hence defined, are unforced oscillations: since $p=0$, no mechanical power arises from a displacement of the membrane, and the following boundary condition guarantees that no thermal power is entering the system

$$
\oint_{\partial V_{f}} \bar{\tau} \partial_{i} \tau n_{i} d S=0
$$

Here, $\partial V_{f}$ is the closed boundary of the fluid volume $V$, made of the pore walls $\partial V$ and contact surfaces with the confining membrane; $n_{i}$ are the components of the unit normal to $\partial V_{f}$, and $\bar{\tau}$ is the field complex conjugate of $\tau$. This condition (C7) is automatically satisfied because the integrand is identically zero on the pore walls and membrane, due to, respectively, boundary conditions (C5) and membrane adiabaticity. From heat equation (C6) we have

$$
-i \omega \rho_{0} C_{p} \int_{V} \bar{\tau} \tau d V=\kappa \int_{V} \bar{\tau} \nabla^{2} \tau d V
$$

Finally, because of the following identity

$$
\bar{\tau} \nabla^{2} \tau=\partial_{i}\left(\bar{\tau} \partial_{i} \tau\right)-\partial_{i} \bar{\tau} \partial_{i} \tau,
$$

and boundary conditions (C7), Eq. (C8) becomes

$$
\omega=-i \nu^{\prime} \frac{\int_{V}|\nabla \tau|^{2} d V}{\int_{V}|\tau|^{2} d V}
$$

Therefore, any pole of $k^{\prime}(\omega)$ lies on the negative imaginary axis.

A pole of $\alpha^{\prime}(\omega)$ means that there exists a nontrivial solution to the microscopic equations (C3)-(C5), having the property that $\langle\tau\rangle=0$, where $\langle\tau\rangle$ is the mean excess temperature in the fluid. Here the membrane is sealed off so that a nonvanishing pressure may arise. As before, no mechanical power is entering the system since the membrane is motionless, and the boundary condition (C7) is fulfilled. From Eq. (C3) we have

$$
-i \omega \rho_{0} C_{p} \int_{V} \bar{\tau} \tau d V=\kappa \int_{V} \bar{\tau} \nabla^{2} \tau d V-i \omega \int_{V} \bar{\tau} p d V
$$

The second integral in the right-hand side is identically zero since $p$ is a constant and $\langle\tau\rangle=0$. Hence, the same result (C10) is obtained, proving that any pole of $\alpha^{\prime}(\omega)$ lies on the negative imaginary axis. To complete the proof and obtain that any zero or branch point of $k^{\prime}(\omega)$ or $\alpha^{\prime}(\omega)$ must occur on the negative imaginary axis, one proceeds exactly as indicated by Johnson et al., ${ }^{10}$ Appendix A.

\footnotetext{
${ }^{1}$ L. L. Beranek, “Acoustic impedance of porous materials,"' J. Acoust. Soc. Am. 13, 248-260 (1942).
} 
${ }^{2}$ C. Zwikker and C. W. Kosten, Sound Absorbing Materials (Elsevier, New York, 1949).

${ }^{3}$ M. R. Stinson, "The propagation of plane sound waves in narrow and wide circular tubes, and generalization to uniform tubes of arbitrary crosssectional shapes,'” J. Acoust. Soc. Am. 89, 550-558 (1991).

${ }^{4} \mathrm{~K}$. Attenborough, "On the acoustic slow wave in air-filled granular media," J. Acoust. Soc. Am. 81, 93-102 (1987).

${ }^{5}$ J. F. Allard, C. Depollier, and A. Aknine, "Acoustical properties of partially reticulated foams with high and medium flow resistance," J. Acoust. Soc. Am. 79, 1734-1740 (1986).

${ }^{6} \mathrm{Y}$. Champoux and M. R. Stinson, "Experimental investigation of models of sound wave propagation in air saturated porous media," J. Acoust. Soc. Am. 88, 121 (1990).

${ }^{7} \mathrm{~K}$. Attenborough, "Models for the acoustical properties of air saturated granular media," Acust. Acta Acust. 1, 213-226 (1993).

${ }^{8}$ Y. Champoux and J. F. Allard, "Dynamic tortuosity and bulk modulus in air-saturated porous media," J. Appl. Phys. 70, 1975-1979 (1991).

${ }^{9}$ D. L. Johnson, J. Koplik, and L. M. Schwartz, "New pore size parameter characterizing transport in porous media," Phys. Rev. Lett. 57, 25642567 (1986).

${ }^{10}$ D. L. Johnson, J. Koplik, and R. Dashen, "Theory of dynamic permeability and tortuosity in fluid saturated porous media," J. Fluid Mech. 176, 379-402 (1987).

${ }^{11}$ P. Leclaire, L. Kelders, W. Lauriks, M. Melon, and B. Castagnède, "Determination of the viscous and the thermal characteristic lengths of plastic foams by ultrasonic measurements in helium and air," J. Appl. Phys. 80, 2009-2012 (1996).

${ }^{12}$ S. Brunauer, P. H. Emmett, and E. Teller, "Absorption of gases in multimolecular layers," J. Am. Chem. Soc. 60, 309-319 (1938).

${ }^{13}$ A. Bardot, B. Brouard, and J. F. Allard, "Frame decoupling at low frequency in thin porous layers saturated by air," J. Appl. Phys. 79, 82238229 (1996).

${ }^{14} \mathrm{M}$. Avellaneda and S. Torquato, "Rigorous link between fluid permeability, electrical conductivity and relaxation times for transport in porous media," Phys. Fluids A 3, 2529-2540 (1991).

${ }^{15} \mathrm{~V}$. Tarnow, "Measurement of sound propagation in glass wool," J. Acoust. Soc. Am. 97, 2272-2281 (1995).

${ }^{16}$ T. Levy and E. Sanchez-Palencia, "Equations and interface conditions for acoustic phenomena in porous media,' J. Math. Anal. Appl. 61, 813-834 (1977).
${ }^{17}$ D. Lafarge, "Propagation du son dans les matériaux poreux à structure rigide saturés par un fluide viscothermique: Définition de paramètres géométriques, analogie electromagnétique, temps de relaxation," $\mathrm{Ph} . \mathrm{D}$. thesis, Université du Maine, Le Mans, France (1993).

${ }^{18}$ M-Y. Zhou and P. Sheng, "First-principles calculations of dynamic permeability in porous media," Phys. Rev. B 39, 12 027-12 039 (1989).

${ }^{19}$ L. M. Schwartz, N. Martys, D. P. Bentz, E. J. Garboczi, and S. Torquato, "Cross-properties relations and permeability estimation in model porous media," Phys. Rev. E 48, 4584-4591 (1993).

${ }^{20}$ C. Straley, A. Matteson, S. Feng, and L. M. Schwartz, "Magnetic resonance, digital analysis, and permeability of porous media," Appl. Phys. Lett. 51, 1146-1148 (1987).

${ }^{21}$ S. Pride, A. Gangi, and F. D. Morgan, "Deriving the equations of motion for porous isotropic media," J. Acoust. Soc. Am. 92, 3278-3290 (1992).

${ }^{22}$ C. Marle, "Ecoulements monophasiques en milieux poreux," Rev. Inst. Français du Pétrole 22, 1471-1509 (1967).

${ }^{23}$ J. F. Allard, Propagation of Sound in Porous Media: Modeling Sound Absorbing Materials (Chapman \& Hall, London, 1993).

${ }^{24}$ A. Craggs and J. G. Hildebrandt, "Effective densities and resistivities for acoustic propagation in narrow tubes," J. Sound Vib. 92, 321-331 (1984).

${ }^{25} \mathrm{~T}$. Yamamoto and A. Turgut, "Acoustic wave propagation through porous media with arbitrary pore size distributions," J. Acoust. Soc. Am. 83, 1744-1751 (1988).

${ }^{26}$ Y. Champoux, "Etude expérimentale du comportement acoustique des matériaux poreux à structure rigide,' Ph.D. thesis, Carleton University, Canada (1991).

${ }^{27}$ V. Tarnow, “Compressibility of air in fibrous materials," J. Acoust. Soc. Am. 99, 3010-3016 (1996).

${ }^{28}$ A. M. Chapman and J. J. L. Higdon, "'Oscillatory Stokes flow in periodic porous media,’ Phys. Fluids A 4, 2099-2116 (1992).

${ }^{29}$ Y. Auregan, private communication.

${ }^{30}$ D. W. Davidson and R. H. Cole, "Dielectric relaxation in glycerol, propylene glycol, and $n$-propanol,', J. Chem. Phys. 19, 1484-1490 (1951).

${ }^{31} \mathrm{~S}$. Torquato and M. Avellaneda, "Diffusion and reaction in heterogeneous media: Pore size distribution, relaxation times, and mean survival time," J. Chem. Phys. 95, 6477-6489 (1991).

${ }^{32}$ L. C. Woods, The Thermodynamics of Fluid Systems (Oxford U.P., New York, 1986). 